\title{
Oxidation of amidoximes with IBX and IBX/ TEAB
}

\author{
Swapnil S. Deshmukh, Sameerana N. Huddar, Dinesh S. Bhalerao, \\ and Krishnacharya G. Akamanchi* \\ Department of Pharmaceutical Sciences \& Technology, Institute of Chemical \\ Technology, Matunga, Mumbai, 400019, India \\ E-mail:kgap@rediffmail.com
}

\begin{abstract}
Biologically important process of oxidation of amidoximes has been investigated using IBX ( $o$ iodoxybenzoic acid) and combination of IBX with TEAB (tetraethylammonium bromide). The reaction proceeds with high \% conversion leading to selective formation of amide and nitrile depending upon the combination of reagents.
\end{abstract}

Keywords: Amidoximes, IBX, TEAB, nitriles, amides

\section{Introduction}

Amidoximes constitute important fragments of many synthetically and biologically valuable compounds. The biological oxidation of L-arginine containing amidoxime $\left[\mathrm{C}\left(\mathrm{NH}_{2}\right)=\mathrm{NOH}\right]$ moiety provides citrulline and nitrogen oxide (NO). ${ }^{1}$ The nitrogen oxide thus generated functions as mediator in regulating diverse physiological processes such as blood pressure, neurotransmission, learning, memory, and immunomodulation. ${ }^{2}$ Synthetic moieties possessing amidoxime function are expected to act as nitrogen oxide donors after oxidative activation in biological system. Based on this hypothesis many amidoximes have been tested on biological systems and were found to show antihypertensive, antiarrhythmic activity. ${ }^{3}$ Both chemical and biomimetic systems have been investigated for oxidation of amidoximes especially with respect to formation of products and the reaction mechanism. A comparative study of chemical vs biomimetic systems was undertaken by Mansuy et al. The studies revealed that the reaction is not smooth and various products formed could be indicators of ensuing oxidation mechanism. ${ }^{4} \mathrm{It}$ has been suggested that the selective formation of nitrile is indicative of successive one electron oxidation whereas the selective formation of amides indicate involvement of peroxidic compounds with a nucleophilic reactivity. The oxidative formation of amide has been reported using hydrogen peroxide, $m$-chloroperbenzoic acid, superoxide anion ${ }^{5 \mathrm{a}}$ and photooxygenation of amidoximate anions $^{5 b}$ whereas nitriles have been formed selectively using $\mathrm{Pb}(\mathrm{OAc})_{4}, \mathrm{AgCO}_{3}$, 
potassium permanganate tetra-butylammonium bromide combination, dibromoisocyanurate and $\mathrm{Pb}_{3} \mathrm{O}_{4}$ in acetic acid. ${ }^{6}$

As part of our ongoing research in the area of oxidative transformations using hypervalent iodine reagents, we became interested in investigation of oxidative transformation of amidoximes using IBX and IBX/TEAB combination. Over the past decade $o$-iodoxybenzoic acid (IBX) has gained dramatic popularity amongst the hypervalent iodine reagents. ${ }^{7,8}$

We have previously reported various newer applications in the field of synthetic chemistry using IBX and IBX-TEAB (tetraethylammonium bromide) combination as oxidizing agents. ${ }^{9}$

IBX as an oxidant readily accepts new heteroatom-based ligands and, thus brings about varieties of oxidative transformations of amines, amides, and their sulfur analogues, ketones, aldehydes, carbamates and oximes derived from urea. ${ }^{8}$

IBX in combination with TEAB brings about oxidative brominations, oxidative bromination followed by dehomologative transformations, oxidative thiocyanations and so on. ${ }^{9 \mathrm{~b}, \mathrm{c}}$ It has been noticed that the course of oxidation of amidoximes changes with the oxidizing agents giving amides or nitriles as the major products. This selectivity shift inspired us to investigate oxidation of amidoximes by using IBX and IBX/TEAB combination.

Table 1. Reaction of benzamidoxime 1a with IBX

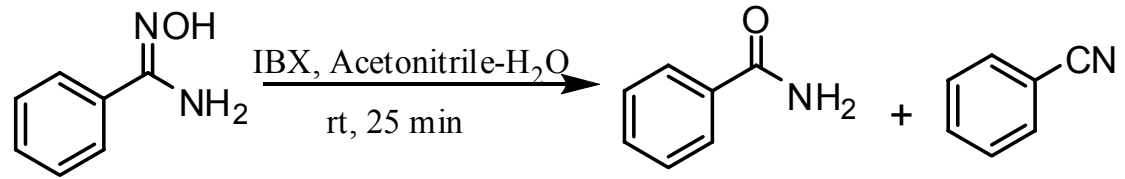

$1 \mathbf{a}$

2a

3a

\begin{tabular}{ccccc}
\hline Entry & Mole ratio (IBX) & $\begin{array}{c}\text { Products } \\
\mathbf{2 a}\end{array}$ & $\begin{array}{c}\text { Yield (\%) } \\
\mathbf{3 a}\end{array}$ & $\begin{array}{c}\text { Unreacted } \\
\mathbf{1 a}(\%)\end{array}$ \\
\hline 1 & 1 & 76 & 7 & 15 \\
$\mathbf{2}$ & $\mathbf{1 . 5}$ & $\mathbf{8 3}$ & 10 & - \\
3 & 2 & 69 & 20 & - \\
4 & 2.5 & 52 & 40 & - \\
\hline
\end{tabular}

\section{Results and Discussion}

\section{Oxidations with IBX}

Investigations were initiated with IBX as oxidant using benzamidoxime 1a as the substrate (Table 1). Reactions were performed in acetonitrile-water mixture at room temperature with equimolar ratio of IBX and benzamidoxime 1a and formation of both amide 2a and nitrile 3a was observed with some amount of starting material remaining unreacted (Table 1, entry1). Upon increasing the reagent/substrate mole ratios, formation of nitrile 3a increases. On the other 
hand, when dry acetonitrile was used the reaction was slow and remained incomplete. A mole ratio of 1: 1.5 (substrate: reagent) which leads to maximum amide formation was chosen for further studies (Table 1, entry 2). Various amidoximes were treated with IBX using the optimized condition and the results are listed in Table 2.

A variety of amidoximes including substituted aromatic, hetero-aromatic, and aliphatic groups were transformed into amides smoothly via this protocol in moderate to good yields.

Alkyl substituted amidoximes also underwent this reaction smoothly (Table 2, entries 6-9). Thiophene and furan rings were stable under the present reaction conditions (Table 2, entries 1112). IBX-mediated oxidations have been explained invoking both ionic as well SET mechanisms. ${ }^{8 a, b, c}$ In the present case formation of amide as major product and in presence of water strongly suggest that the reaction might be proceeding predominantly by ionic mechanism as shown in Scheme 1.

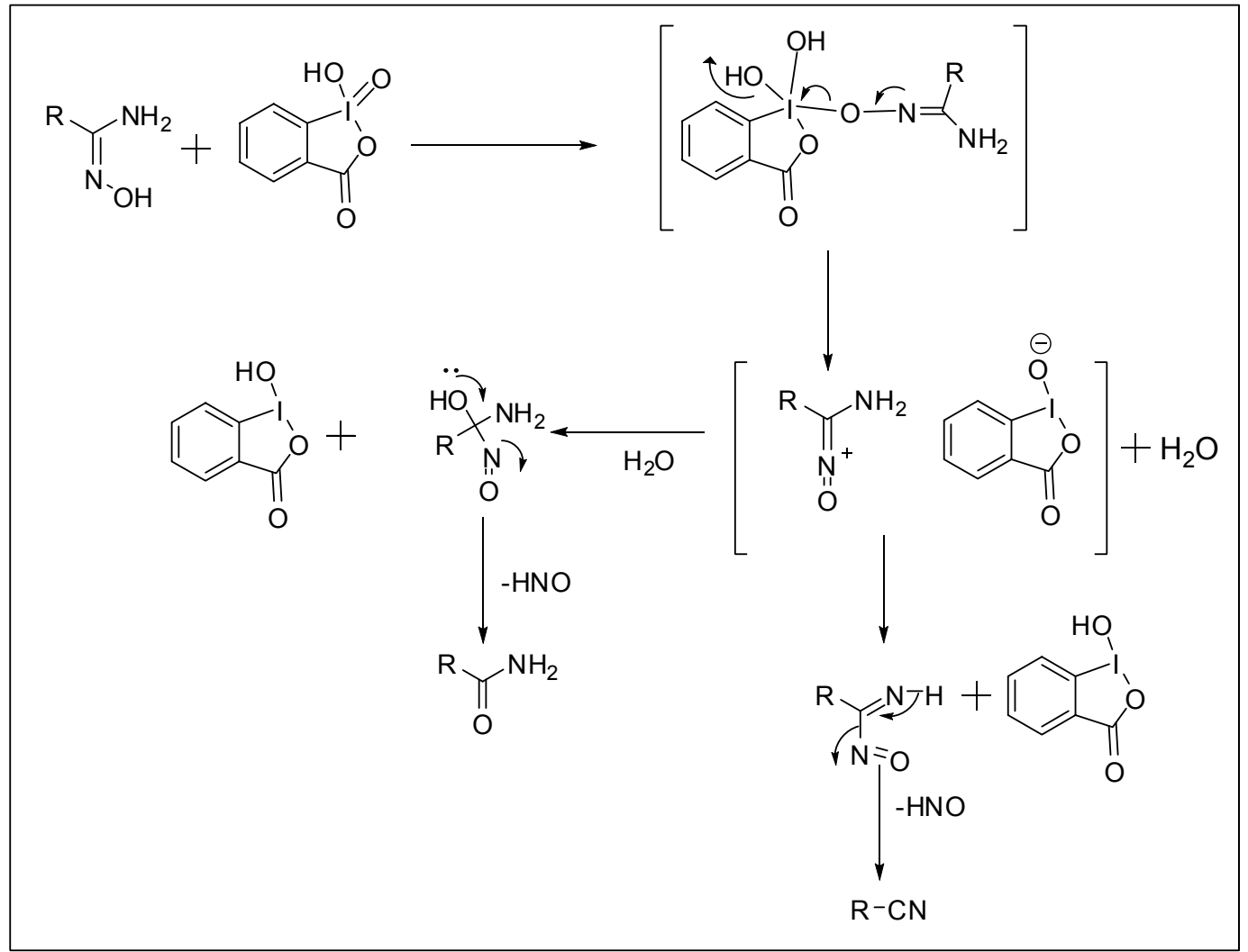

Scheme 1. Plausible mechanism of formation of amide and nitrile with IBX 
Table 2. Oxidation of amidoximes by IBX

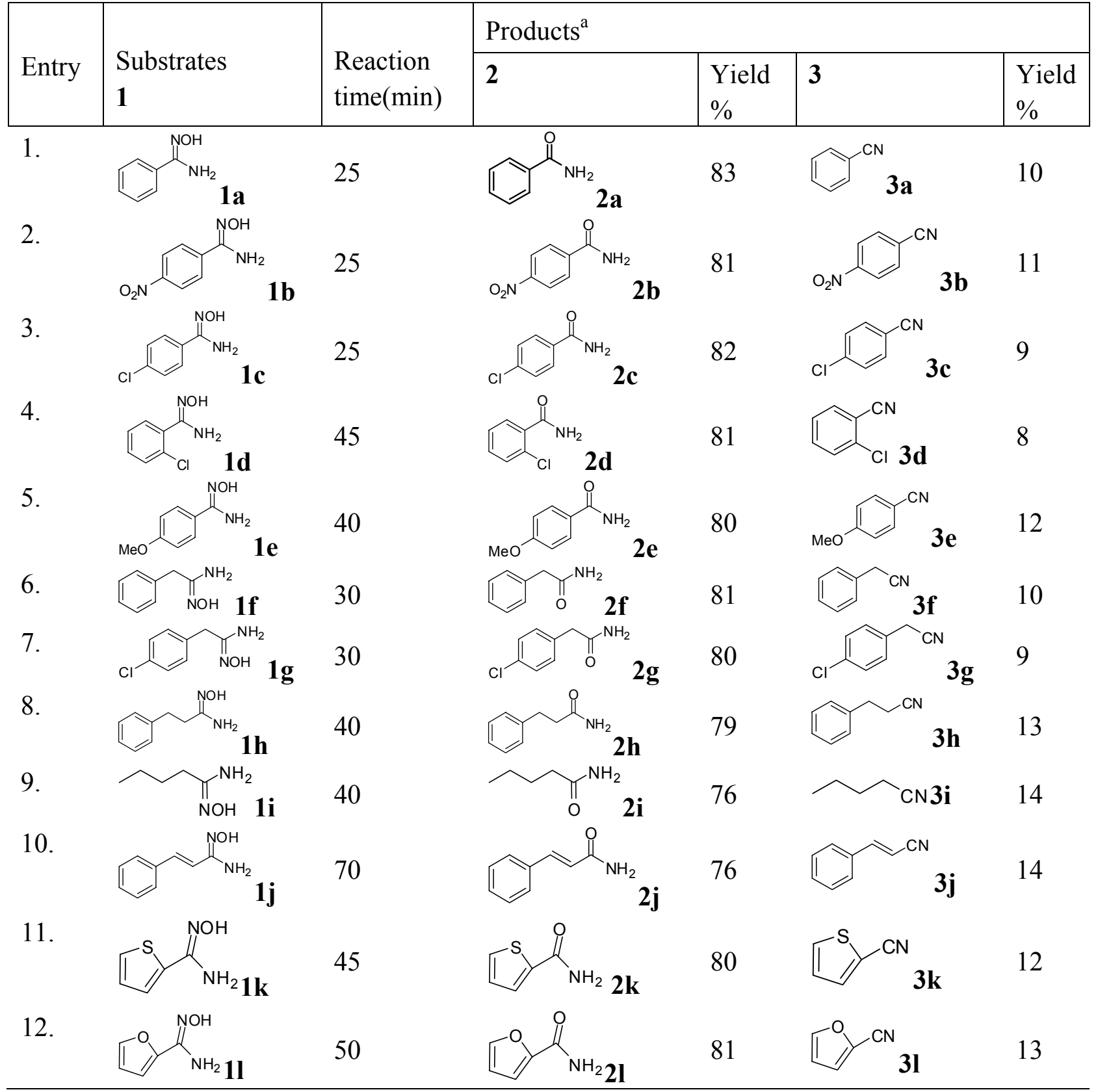

${ }^{\text {a }}$ Products were characterized by ${ }^{1} \mathrm{H}$ NMR and IR analysis data, yields presented are after column chromatography.

\section{Oxidations with IBX/TEAB}

The IBX/TEAB combination has been investigated and reactions were carried out with benzamidoxime 1a as model substrate (Table 3). It is interesting to note that with this oxidation system nitrile 3a was formed as the major product. 
The mole ratio study revealed that a mole ratio of 1:2:2 of amidoxime:IBX: TEAB is optimum to attain higher yield of nitrile 3a (Table 3, entry 4). Under the standardized reaction conditions various amidoximes were reacted and the results are summarized in Table 4. It is observed that reactivity pattern was similar to that with the oxidations using IBX.

Table 3. Reaction of benzamidoxime 1a with IBX/TEAB

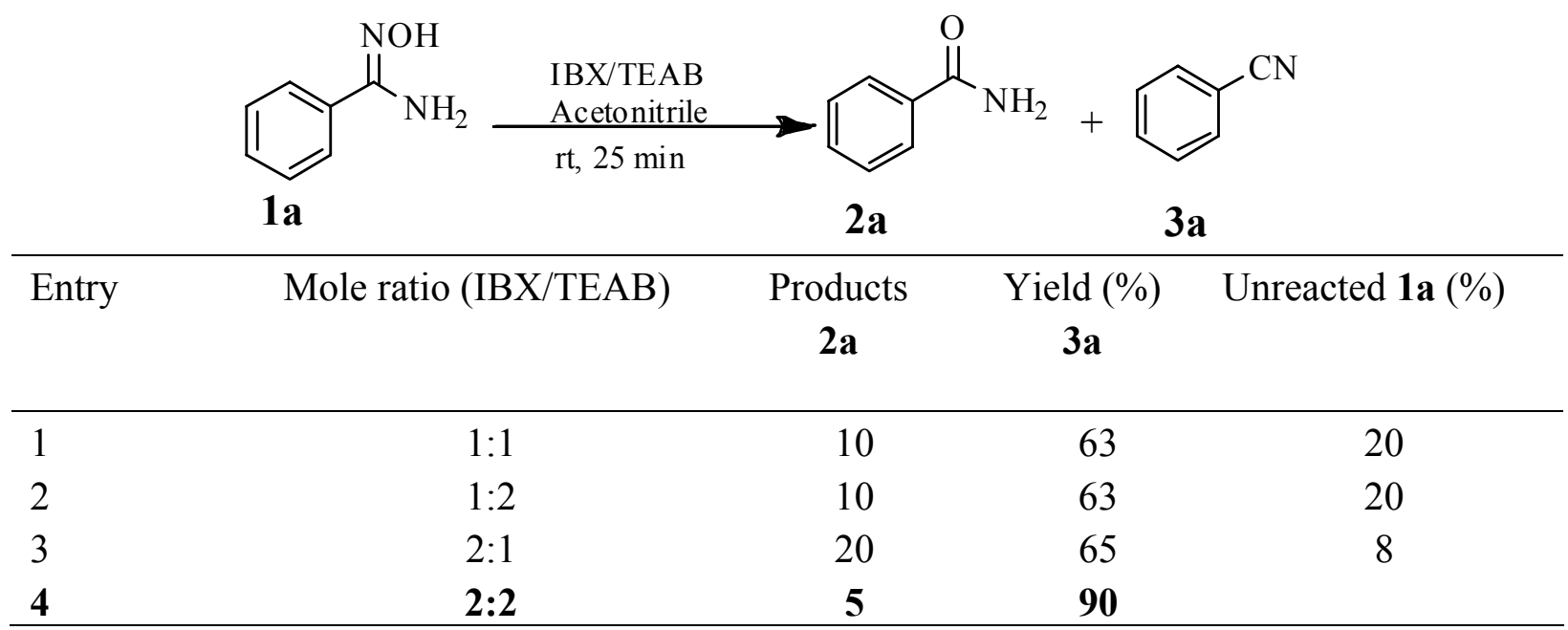

In case of cinnamyl amidoxime the double bond was unaffected and the reaction was comparatively slower (Table 4, entry 10). Thiophene and furan rings were stable under the present reaction conditions (Table 4, entries 11-12). To account for the role of TEAB and formation of very high yields of nitriles an alternative mechanism is suggested as shown in Scheme 2.

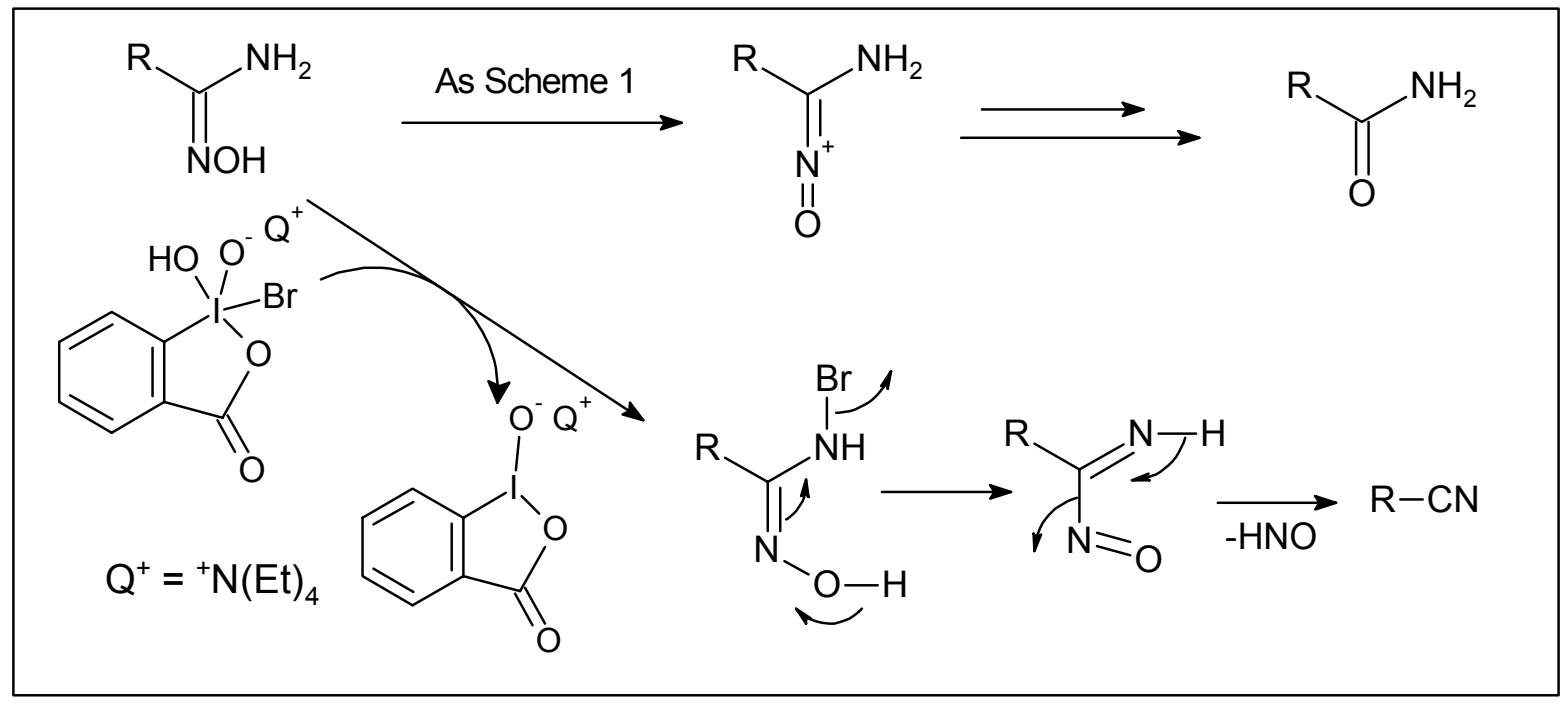

Scheme 2. Plausible mechanism for formation of nitrile and role of TEAB. 
Table 4. IBX/ TEAB mediated oxidative conversion of amidoxime to nitriles

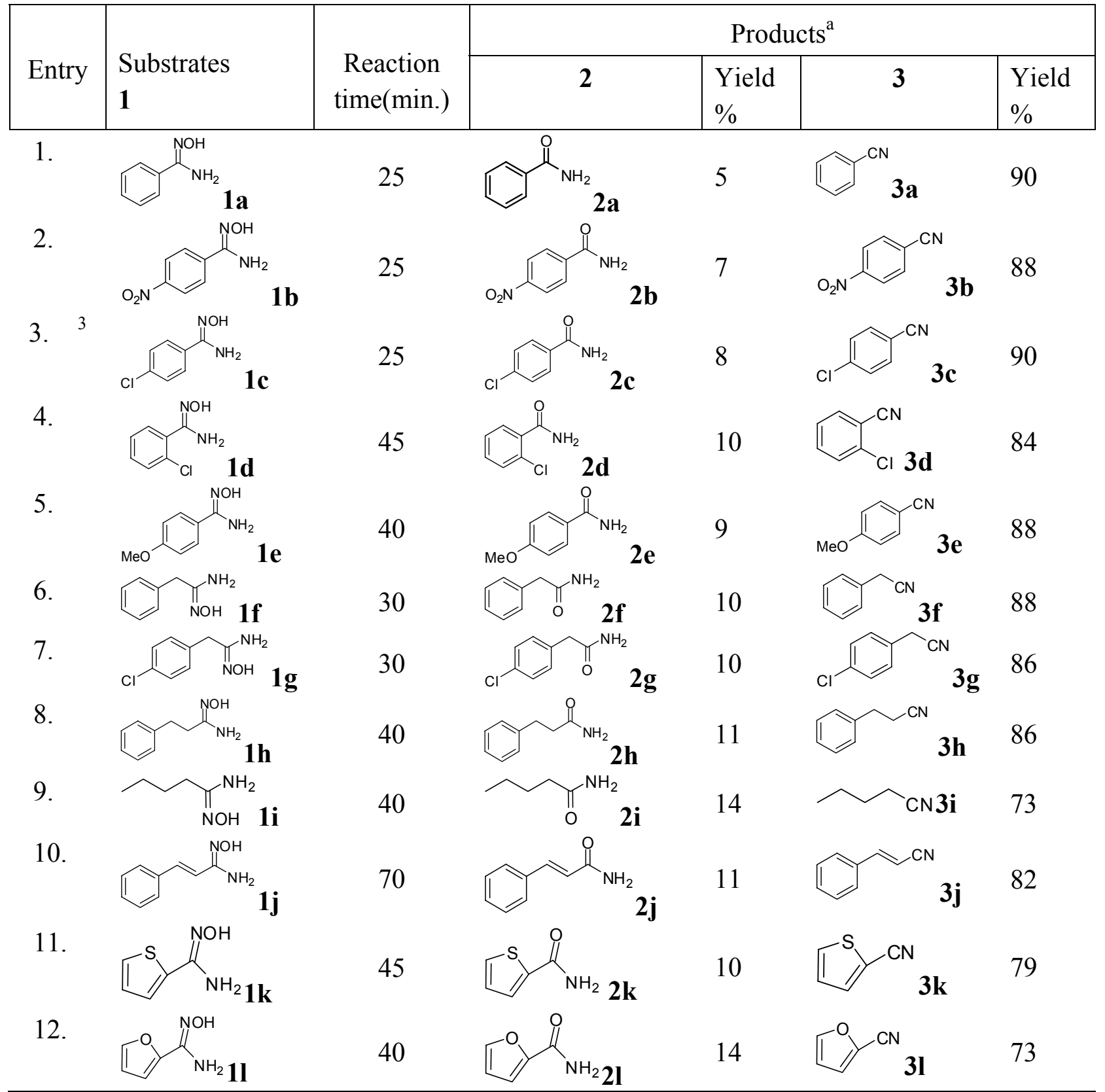

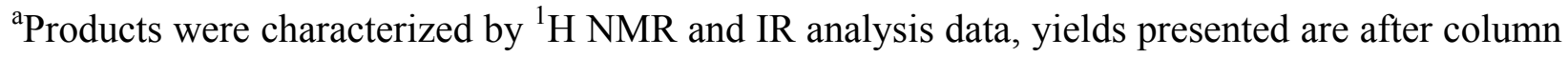
chromatography.

\section{Conclusions}

In conclusion, the oxidation of amidoximes with IBX and IBX/TEAB combination gives different results. Reaction with IBX gives amides as the major product where as reaction with 
IBX/TEAB combination gives nitrile as the major product. The substituents influence the reaction rate but with no significant effect on selectivity. These results could be valuable for mechanistic investigations in chemistry of hypervalent iodine(V) reagents.

\section{Experimental Section}

General. ${ }^{1} \mathrm{H}$ NMR spectra were recorded on JEOL MY-60 operating at $60 \mathrm{MHz}$ and JEOL-FTNMR operating at $300 \mathrm{MHz}$ instruments, chemical shifts are expressed in parts per million downfield from TMS in $\delta$ units. IR spectra were recorded on FTIR RX1 Perkin-Elmer instrument. Melting points were determined with Veego melting point apparatus having stirred paraffin bath. Silica gel 60-120 was used for column chromatography and Thin Layer Chromatography (TLC) was performed using Merck Silica gel $60 \mathrm{~F}_{254}$ Plates. All the amidoximes were prepared by the literature procedures. ${ }^{[3,10]}$

\section{General experimental procedure for oxidation of amidoximes with IBX}

To a stirred suspension of IBX $(7.5 \mathrm{mmol})$ in aqueous acetonitrile $\left(\mathrm{CH}_{3} \mathrm{CN} 15 \mathrm{ml}+\right.$ water $\left.2 \mathrm{ml}\right)$ was added amidoxime $(5 \mathrm{mmol})$. The reaction mixture was stirred at room temperature for the period mentioned. Acetonitrile was removed under reduced pressure and the resultant residue was extracted with ethyl acetate $(2 \times 25 \mathrm{ml})$. The organic layer was washed with $10 \%$ sodium bisulfite solution $(25 \mathrm{ml})$, saturated sodium carbonate $(25 \mathrm{ml})$ and brine $(25 \mathrm{ml})$, dried over anhydrous sodium sulfate and concentrated under reduced pressure to give crude product. Pure product was isolated after column chromatography (silica gel mesh size 60-120, eluent ethyl acetate: $n$-hexane $3: 4)$.

\section{General experimental procedure for oxidation of amidoximes with IBX/TEAB}

To a stirred suspension of IBX $(11 \mathrm{mmol})$ in $\mathrm{CH}_{3} \mathrm{CN}(20 \mathrm{ml})$ was added TEAB $(11 \mathrm{mmol})$. To the resultant yellow suspension substrate $(5 \mathrm{mmol})$ was added in one portion after $5 \mathrm{~min}$. The reaction mixture was stirred at room temperature for the specified period. Acetonitrile was removed under reduced pressure and resultant residue was washed with $25 \mathrm{ml}$ of ethyl acetate. The organic layer was washed with $10 \%$ sodium bisulfite solution $(25 \mathrm{ml})$, saturated sodium carbonate $(25 \mathrm{ml})$ and brine $(25 \mathrm{ml})$. The organic layer was dried over anhydrous sodium sulfate and concentrated under reduced pressure to give crude product. Pure product was isolated after column chromatography (silica gel mesh size 60-120, eluent ethyl acetate: $n$-hexane 1:4).

\section{Spectral data of selected amides}

Benzamide 2a. Solid, Mp $124{ }^{\circ} \mathrm{C}$ (lit. ${ }^{11 \mathrm{a}} 122-126{ }^{\circ} \mathrm{C}$ ). IR (KBr): 3500, 3200, $1670 \mathrm{~cm}^{-1} .{ }^{1} \mathrm{H}$ $\operatorname{NMR}\left(60 \mathrm{MHz}, \mathrm{CDCl}_{3}\right): \delta=7.40-7.81(5 \mathrm{H}, \mathrm{m}), 5.90(2 \mathrm{H}, \mathrm{br} \mathrm{s})$.

4-Nitrobenzamide 2b. Solid, Mp $199-201{ }^{\circ} \mathrm{C}$ (lit. $\left.{ }^{11 \mathrm{~b}} 201{ }^{\circ} \mathrm{C}\right)$. IR (KBr): $3520,3230,1668 \mathrm{~cm}^{-1}$ ${ }^{1} \mathrm{H}$ NMR $\left(300 \mathrm{MHz}, \mathrm{CDCl}_{3}\right): \delta=8.30(2 \mathrm{H}, \mathrm{d}, J=8.74 \mathrm{~Hz}), 7.96(2 \mathrm{H}, \mathrm{d}, J=8.75 \mathrm{~Hz}), 6.00(2 \mathrm{H}$, br s). 
4-Methoxybenzamide 2e. Solid, $\mathrm{Mp} 166-167^{\circ} \mathrm{C}$ (lit. ${ }^{12 \mathrm{a}} 165-167^{\circ} \mathrm{C}$ ). IR (KBr): 3500, 3400, $1680 \mathrm{~cm}^{-1} .{ }^{1} \mathrm{H}$ NMR $\left(300 \mathrm{MHz}, \mathrm{CDCl}_{3}\right): \delta=7.76(2 \mathrm{H}, \mathrm{d}, J=8.70 \mathrm{~Hz}$ ) $), 6.90(2 \mathrm{H}, \mathrm{d}, J=9.00$ $\mathrm{Hz}), 5.86$ (2H, br s), $3.83(3 \mathrm{H}, \mathrm{s})$.

Phenylacetamide 2f. Solid, Mp $155{ }^{\circ} \mathrm{C}$ (lit. $\left.{ }^{12 \mathrm{~b}} 157-158{ }^{\circ} \mathrm{C}\right)$. IR (KBr): $3500,3200,1670 \mathrm{~cm}^{-1}$. ${ }^{1} \mathrm{H}$ NMR $\left(60 \mathrm{MHz}, \mathrm{CDCl}_{3}\right) \delta=7.23-7.33(5 \mathrm{H}, \mathrm{m}), 7.21(2 \mathrm{H}, \mathrm{br} \mathrm{s}), 3.85(2 \mathrm{H}, \mathrm{s})$.

3-Phenylpropanamide 2h. Solid, Mp $105{ }^{\circ} \mathrm{C}$ (lit. ${ }^{12 \mathrm{c}} 105{ }^{\circ} \mathrm{C}$ ). IR (KBr): $3500,3200,1669 \mathrm{~cm}^{-1}$. ${ }^{1} \mathrm{H}$ NMR $\left(60 \mathrm{MHz}, \mathrm{CDCl}_{3}\right): \delta=7.29-7.40(5 \mathrm{H}, \mathrm{m}), 7.03(2 \mathrm{H}, \mathrm{s}), 2.80(2 \mathrm{H}, \mathrm{t}, J=7.4 \mathrm{~Hz}), 2.57(2 \mathrm{H}$, $\mathrm{t}, J=7.4 \mathrm{~Hz}$ ).

Pentanamide 2i. Solid, Mp 102-104 ${ }^{\circ} \mathrm{C}$ (lit. ${ }^{12 \mathrm{~d}} 103-106{ }^{\circ} \mathrm{C}$ ). IR (KBr): $3500,3200,1670 \mathrm{~cm}^{-1}$. ${ }^{1} \mathrm{H}$ NMR $\left(300 \mathrm{MHz}, \mathrm{CDCl}_{3}\right) \delta=5.88(2 \mathrm{H}$, br s), $2.18(2 \mathrm{H}, \mathrm{t}), 1.60(2 \mathrm{H}, \mathrm{m}), 1.30(2 \mathrm{H}, \mathrm{m}), 0.89$ $(3 \mathrm{H}, \mathrm{t})$.

Cinnamamide 2j. Solid, Mp $145{ }^{\circ} \mathrm{C}$ (lit. ${ }^{12 \mathrm{~b}} 147^{\circ} \mathrm{C}$ ). IR (KBr): 3372, 3165, $1665 \mathrm{~cm}^{-1}$. ${ }^{1} \mathrm{H}$ NMR $\left(60 \mathrm{MHz}, \mathrm{CDCl}_{3}\right): \delta=7.23-7.33(5 \mathrm{H}, \mathrm{m}), 7.21(2 \mathrm{H}, \mathrm{s}), 3.85(2 \mathrm{H}, \mathrm{s})$.

Thiophene-2-carbamide 2k. Solid, Mp $178{ }^{\circ} \mathrm{C}$ (lit. ${ }^{12 \mathrm{e}} 180{ }^{\circ} \mathrm{C}$ ). IR (KBr): 3400, 3190, 1660, $1630 \mathrm{~cm}^{-1} .{ }^{1} \mathrm{H}$ NMR $\left(300 \mathrm{MHz}, \mathrm{CDCl}_{3}\right): \delta=7.52-7.49(2 \mathrm{H}, \mathrm{m}), 7.10-7.09(1 \mathrm{H}, \mathrm{m}), 5.76(2 \mathrm{H}, \mathrm{br}$ s).

Furan-2-carbamide 21. Solid, Mp $140{ }^{\circ} \mathrm{C}$ (lit. ${ }^{12 \mathrm{a}} 140-141{ }^{\circ} \mathrm{C}$ ). IR (KBr): 3390, 3160, 1660, $1630 \mathrm{~cm}^{-1} .{ }^{1} \mathrm{H}$ NMR $\left(300 \mathrm{MHz}, \mathrm{CDCl}_{3}\right): \delta=7.45-7.41(1 \mathrm{H}, \mathrm{m}), 7.14-7.09(1 \mathrm{H}, \mathrm{m}), 6.50-6.44$ $(1 \mathrm{H}, \mathrm{m}), 6.18(2 \mathrm{H}$, br s).

\section{Spectral data of selected nitriles}

Benzonitrile 3a. Oil (Lit. ${ }^{13}$ Oil). IR (neat): $2230 \mathrm{~cm}^{-1} .{ }^{1} \mathrm{H}$ NMR $\left(60 \mathrm{MHz}, \mathrm{CDCl}_{3}\right): \delta=7.60-7.46$ $(5 \mathrm{H}, \mathrm{m})$.

4-Nitrobenzonitrile 3b. Solid, Mp 140-142 ${ }^{\circ} \mathrm{C}$ (lit. ${ }^{13} 142{ }^{\circ} \mathrm{C}$ ). IR (KBr): $2231 \mathrm{~cm}^{-1}$. ${ }^{1} \mathrm{H}$ NMR $\left(300 \mathrm{MHz}, \mathrm{CDCl}_{3}\right): \delta=8.38(2 \mathrm{H}, \mathrm{d}, J=8.9 \mathrm{~Hz}), 7.92(2 \mathrm{H}, \mathrm{d}, J=8.9 \mathrm{~Hz})$.

4-Methoxybenzonitrile 3e. Solid, $\mathrm{Mp} 55-56{ }^{\circ} \mathrm{C}$ (lit. ${ }^{13} 55-57{ }^{\circ} \mathrm{C}$ ). IR (neat): $2217 \mathrm{~cm}^{-1}$. ${ }^{1} \mathrm{H}$ NMR $\left(300 \mathrm{MHz}, \mathrm{CDCl}_{3}\right): \delta=7.77(2 \mathrm{H}, \mathrm{d}, J=8.9 \mathrm{~Hz}), 6.92(2 \mathrm{H}, \mathrm{d}, J=8.9 \mathrm{~Hz}), 3.83(3 \mathrm{H}, \mathrm{s})$.

Phenylacetonitrile 3f. Oil (Lit. ${ }^{12 \mathrm{c}}$ Oil). IR (neat): $2222 \mathrm{~cm}^{-1} .{ }^{1} \mathrm{H} \mathrm{NMR}\left(60 \mathrm{MHz}, \mathrm{CDCl}_{3}\right): \delta=$ 7.62-7.56 (5H, m), $3.23(2 \mathrm{H}, \mathrm{s})$.

3-Phenylpropanenitrile 3h. Oil (Lit. ${ }^{12 \mathrm{c}}$ Oil). IR (neat): $2248 \mathrm{~cm}^{-1}$. ${ }^{1} \mathrm{H} \mathrm{NMR}\left(60 \mathrm{MHz}, \mathrm{CDCl}_{3}\right)$ : $\delta=7.23-7.25(5 \mathrm{H}, \mathrm{m}), 3.62-2.45(4 \mathrm{H}, \mathrm{m})$.

Pentanenitrile 3i. Oil (Lit. ${ }^{12 \mathrm{c}}$ Oil). IR (neat): $2246 \mathrm{~cm}^{-1} .{ }^{1} \mathrm{H}$ NMR $\left(300 \mathrm{MHz}, \mathrm{CDCl}_{3}\right): \delta=1.87$ $(2 \mathrm{H}, \mathrm{t}, J=7.1 \mathrm{~Hz}), 1.66(2 \mathrm{H}, \mathrm{m}), 1.31(2 \mathrm{H}, \mathrm{m}), 0.88(3 \mathrm{H}, \mathrm{t}, J=7.1 \mathrm{~Hz})$.

Cinnamonitrile 3j. Oil (lit. $\left.{ }^{12 \mathrm{c}} 20-21{ }^{\circ} \mathrm{C}\right)$. IR (neat): $2216 \mathrm{~cm}^{-1} .{ }^{1} \mathrm{H}$ NMR $\left(300 \mathrm{MHz}, \mathrm{CDCl}_{3}\right): \delta=$ $7.41(1 \mathrm{H}, \mathrm{d}, J=16.7 \mathrm{~Hz}), 7.38-.7 .48(5 \mathrm{H}, \mathrm{m}), 5.89(1 \mathrm{H}, \mathrm{d}, J=16.7 \mathrm{~Hz})$.

2-Cyanothiophene 3k. Oil (Lit. ${ }^{13}$ Oil).. IR (Neat): $2228 \mathrm{~cm}^{-1} .{ }^{1} \mathrm{H}$ NMR $\left(300 \mathrm{MHz}, \mathrm{CDCl}_{3}\right): \delta=$ $7.97(1 \mathrm{H}, \mathrm{dd}, J=5.0$ and $3.8 \mathrm{~Hz}) 7.64(1 \mathrm{H}, \mathrm{d}, J=3.8 \mathrm{~Hz}), 7.61(1 \mathrm{H}, \mathrm{d}, J=5.0 \mathrm{~Hz})$.

2-Cyanofuran 31. Oil (Lit. ${ }^{[12 \mathrm{c}}$ Oil). IR (Neat): $2230 \mathrm{~cm}^{-1} .{ }^{1} \mathrm{H}$ NMR $\left(60 \mathrm{MHz}, \mathrm{CDCl}_{3}\right): \delta=8.03$ $(1 \mathrm{H}, \mathrm{d}, J=1.6 \mathrm{~Hz}), 7.15(1 \mathrm{H}, \mathrm{d}, J=3.62), 6.7(1 \mathrm{H}, \mathrm{dd}, J=3.6$ and $1.6 \mathrm{~Hz})$. 


\section{Acknowledgements}

For financial assistance S.S.D. thanks the Council of Scientific and Industrial Research (CSIR), India and S.N.H thanks Narotam Sekhsaria Foundation, Mumbai. We are also thankful to M/S Omkar Chemicals in Badalapur, Thane, India, for their generous gift of IBX.

\section{References}

1. (a) Feldman, P. L.; Griffith, O.W.; Stuehr, D. J. Chem. Eng. News 1993, 71, 26. (b) Fukuto, J. M.; Komori, Y. Ann. Rep. Med. Chem. 1994, $29,83$.

2. Moncada, S.; Palmer, R. M. J.; Higgs, E. A. Pharmacol. Rev. 1991, 43, 109.

3. Nicolaides, D. N.; Varella, E. A The Chemistry of Acid Derivatives, Vol. 2, John Wiley: New York, 1992; p 876.

4. Goff, S. V.; Boucher, J.; Mansuy, D. Chimie bioorganique et therapeutique,2000, 3, 785.

5. (a) Sennequier, N.; Boucher, J.; Battioni, P.; Mansuy, D. Tetrahedron Lett. 1995, 36, 6059. (b) Ocal, N.; Erden, I. Tetrahedron Lett. 2001, 42, 4765.

6. Yarovenko, V. N.; Krayushkin, M. M.; Lysenko, O. V.; Kustov, L. M.; , Zavarzin, I. V. Russian Chemical Bulletin, 1994, 43, 402.

7. (a) Zhdankin, V. V.; Stang, P. J. Chem. Rev. 2008, 108, 5299. (b) Ladziata, U.; Zhdankin, V. V. Arkivoc 2006, (ix), 26. (c) Zhdankin, V. V. Arkivoc 2009, (i), 1. (d) Zhdankin, V. V.; Stang, P. J. Chem. Rev. 2002, 102, 2523.

8. (a) Nicolaou, K. C.; Montagnon, T.; Baran, P. S. Angew. Chem. Int. Ed. 2002, 41, 993. (b) Nicolaou, K. C.; Gray, D. L. F.; Montagnon, T.; Harrison, S. T. Angew. Chem. Int. Ed. 2002, 41, 996. (c) Nicolaou, K. C.; Mathison, C. J. N.; Montagnon, T. Angew. Chem. Int. Ed. 2003, 42, 4077.

9. (a) Shukla, V. G.; Salgaonkar, P. D.; Akamanchi, K. G. J. Org. Chem. 2003, 68, 5422. (b) Bhalerao, , D. S.; Mahajan, U. S.; Chaudhari, K. H.; Akamanchi, K. G. J. Org. Chem. 2007, 72, 662. (c) Bellale, E.V.; Bhalerao, D. S.; Akamanchi, K. G. J. Org. Chem. 2008, 73, 9473. (d) Chaudhari, S.S.; Akamanchi, K. G. Tetrahedron Lett. 1998, 39, 3209.

10. Eloy, F.; Lenaers, R. Chem Rev. 1962, 62, 155.

11. (a). Reed, K. L.; Gupton, J. T.; Solarz, T. L. Synth. Commun. 1990, 20, 563. (b) Field, L.; Hughmart, P. B.; Shumaker, S. H.; Marshall, W. S. J. Am. Chem. Soc. 1961, 83, 1983.

12. (a) Matsuura, I.; Ueda, T.; Murakami, N.; Nagai, S. I.; Sakakibara, J. J. Chem. Soc., Perkin Trans. 1 1991, 2821. (b) CRC, Hand Book of Tables for Organic Compound Identification $54^{\text {th }}$ and $80^{\text {th }}$ (c) Dictionary of Organic Compounds, Sixth Edition, Chapman and Hall Electronic Publishing house, London, 1996. (d) Blanchette, J. A.; Brown, E. V. J. Am. Chem. Soc. 1952, 74, 2098. (e) Grochowski, E.; Jurczak, J. J.Org. Chem. 1978, 43, 2541.

13. Iida, S.; Ohmura, R.; Togo, H. Tetrahedron 2009, 65, 6257. 